\title{
Temporomandibular disorders in patients with schizophrenia using antipsychotic agents: a discussion paper
}

This article was published in the following Dove Press journal:

Drug, Healthcare and Patient Safety

10 March 2014

Number of times this article has been viewed

\author{
Arão Nogueira de Araújo' \\ Marion Alves do \\ Nascimento' \\ Eduardo Pondé de Sena ${ }^{1,2}$ \\ Abrahão Fontes Baptista ${ }^{3,4}$ \\ 'Postgraduate Program in Interactive \\ Processes of Organs and Systems, \\ ${ }^{2}$ Department of Pharmacology, \\ Institute of Health Sciences, \\ ${ }^{3}$ Department of Biomorphology, \\ Institute of Health Sciences, \\ ${ }^{4}$ Postgraduate Program in Medicine \\ and Health, Federal University of \\ Bahia, Salvador, Brazil
}

\begin{abstract}
Patients with psychiatric problems show a tendency to develop temporomandibular disorders (TMD). Particularly, patients with schizophrenia are quite likely to have signs and symptoms of TMD due to the impairment of their oral health, the use of antipsychotic drugs, and other general health problems. In nonschizophrenic populations, TMD have been considered as the main cause of nondental pain in the orofacial region, involving mechanisms associated with changes in masticatory activity at the cortical and neuromuscular levels. Individuals with schizophrenia do not usually complain of pain, and TMD is misdiagnosed in this population. In this paper, we aimed to review the clinical aspects of TMD in people with schizophrenia on antipsychotic drug therapy.
\end{abstract}

Keywords: schizophrenia, temporomandibular joint, pain, antipsychotic agents

\section{Introduction}

Schizophrenia is a severe, chronic, and debilitating neuropsychiatric disorder that involves a profound psychopathology disruption of cognition, emotion, perception, and other aspects of behavior. It is an illness that compromises the effective functioning of the individual in social, occupational, and daily life. ${ }^{1}$ It has a worldwide prevalence of approximately $1 \%$, begins most commonly in individuals who are $15-35$ years old, and affects men and women equally. Its management involves the use of antipsychotic drugs, somatic treatment, and psychosocial therapies. ${ }^{2-4}$

Like other psychiatric problems, schizophrenia is associated with a high risk for the development of temporomandibular disorders (TMD), perhaps due to both the effect of prolonged use of antipsychotic medications and inherent conditions associated with the illness itself., ${ }^{2,5}$ Furthermore, individuals with schizophrenia are generally not concerned with their own dental health, tending to present with inadequate oral hygiene, which can contribute to the worsening of a number of issues related to general health. ${ }^{6}$ Also, there is an increased incidence of periodontal disease, erosion, cervical abrasion, and oral cancer due to excessive smoking observed in individuals with schizophrenia. ${ }^{1,7}$

Commonly, in TMD, pain arises as a major symptom that drives patients to search for help. ${ }^{2}$ However, individuals who are suffering from mental disorders - such as schizophrenia - appear less sensitive to somatic pain. ${ }^{8}$ Therefore, in the assessment of TMD in patients with schizophrenia, the most common clinical signs are articular sounds. ${ }^{9}$

Particularly, the location, intensity, and quality of the pain can be aberrantly expressed in schizophrenia. In addition, patients with schizophrenia show very 
limited responses of self-report of pain. It is observed that these patients may be less sensitive to chronic pain, but they are more sensitive to acute pain. ${ }^{10}$ This can limit the understanding of the patients' pain complaint by their families and caregivers. ${ }^{11}$ Discussing TMD in this specific population may be important to help health practitioners identify these disorders and encourage researchers to find new ways of approach.

\section{Conceptualization of temporomandibular problems}

TMD is a terminology that brings together a number of clinical problems that involve the masticatory muscles, the temporomandibular joint, and other associated structures of the stomatognathic system. ${ }^{12,13}$ Also called "craniomandibular disorders", they are considered to be multifactorial and are often reported as affecting young and middleaged adults (appearing in those in the 20-50 age range), with greater prevalence in women than in men. Signs and symptoms of these clinical conditions involve limitation of mouth opening, presence of pain in the masseter and temporal muscle regions, and pain, crackles, and crashes in the temporomandibular joint. ${ }^{12,14-17}$ Their etiology is influenced by degenerative or traumatic injury, musculoskeletal problems, malocclusion, parafunctional habits, and psychosocial factors. ${ }^{13,15,18}$

In general, symptoms of TMD fluctuate over time and affect anatomical, neuromuscular, and psychological aspects. ${ }^{2,17}$ Therefore, TMD are significantly associated with masticatory muscle tension, clenching, grinding, and other deleterious habits that have been commonly related to changes in the central nervous system. ${ }^{17}$ Also, they are commonly associated with symptoms affecting the head and neck, such as headache, cervical spine dysfunction and auditory symptoms. ${ }^{15}$ Factors that increase muscle tension in the orofacial region tend to contribute significantly to the development and aggravation of TMD. Motor dysfunction, for example, can lead to overactivity of the masticatory muscles, increasing their anaerobic metabolism. As a consequence, the release of lactic acid, a known irritant to the nerve terminals, may trigger the sensation of pain. ${ }^{14}$

Among the various factors that contribute to the cause and maintenance of TMD, those resulting from psychosocial processes deserve special attention. This is because it is quite common to find in psychiatric populations greater prevalence of problems related to the temporomandibular joint than in other populations. In addition, mental disorders and TMD seem to share some of the same comorbidities. Patients with
TMD and mental disorders suffer severe reductions of aspects related to quality of life. ${ }^{12}$

\section{Prevalence of temporomandibular problems}

Diagnosis, treatment, etiology, and pathogenesis of TMD present many controversies. For a long time, TMD were reported erroneously as a single syndrome. As TMD cannot be considered a single syndrome, different treatments are supposed to play a role in their therapeutic management. ${ }^{19}$ Therefore, some researchers have emphasized that TMD consist in multiple disorders with similar signs and symptoms that require different approaches. ${ }^{16,20}$ Most TMD cases involve disc displacement, muscular disorders, degenerative changes, or internal derangements of the temporomandibular joint. $^{21}$ Thus, patients with TMD tend to experience pain, limited movement, or asymmetric jaw and temporomandibular joint sounds. ${ }^{16}$

Inflammatory, traumatic, infectious, congenital, developmental, and neoplastic diseases are likely to affect the temporomandibular joint and promote the emergence of TMD. ${ }^{16}$ From the epidemiological point of view, the distribution of TMD varies, depending upon the elected samples and method of evaluation employed..$^{9}$ According to Al-Jundi et al, ${ }^{22}$ differences in estimated prevalence of TMD in the literature probably reflect different conceptions of these disorders, as well as other methodologic influences, such as the time when each study of prevalence occurred and the settings where they were performed. It is estimated that TMD affect more than $25 \%$ of the general population, ${ }^{15}$ and at least $6 \%$ of the people suffer from chronic orofacial pain arising from such disorders. ${ }^{23}$

The diagnosis of TMD requires patients' complaints and takes into consideration: the evaluation of the jaw muscles, bone, and cartilage joint structures; soft tissue joint structures, including the synovial membrane and articular disc, jaw, and joint function; and analysis of painful disorder, according to specific patient behavior. ${ }^{24}$ The fact that psychiatric populations have more positive responses to joint and muscle palpation does not necessarily make them patients with TMD. ${ }^{25}$

Particularly, people with schizophrenia have a high-risk of developing TMD, due to the prolonged experience of emotional distress and the continued use of antipsychotic medications. Also, lifestyle habits and conditions, including socioeconomic factors and the inability to maintain self-care may further contribute to the increase of such risk. ${ }^{2}$ In a casecontrol study, Velasco-Ortega et $\mathrm{al}^{9}$ found the prevalence of signs of TMD in $32 \%$ of patients with schizophrenia. In turn, Gurbuz et $\mathrm{al}^{2}$ observed the prevalence of any TMD sign in 
$83.7 \%$ of patients with schizophrenia, and more than one sign of TMD in $38.6 \%$ of these patients.

Patients with chronic TMD commonly report poor quality of sleep, depressive symptoms, and loss of energy. ${ }^{15}$ However, few studies have examined the prevalence of mental disorders and their association with pain in the temporomandibular joint. Pain seems to be underestimated in psychotic patients, such as those affected by schizophrenia. ${ }^{2}$

Indeed, in samples of patients with chronic pain, there is a lower prevalence of individuals with schizophrenia as compared to people with other psychiatric disorders. ${ }^{26}$ However, patients with schizophrenia have a high risk of developing health problems and, with the exception of rates of suicides and accidents, they also have a life expectancy $20 \%$ lower than the general population. ${ }^{1}$

\section{Antipsychotics agents and adverse effects}

TMD are considered within the subclassification of musculoskeletal disorders. ${ }^{25}$ Therefore, they must be differentially diagnosed from the orofacial movement disorders that are centrally mediated pathological conditions, involving the basal ganglia and their communication with other brain areas. Drug-induced extrapyramidal symptoms, for example, can be presented as movement disorders that provoke orofacial contractions of the tongue, lips, jaw muscles, and temporomandibular joint displacement. ${ }^{27}$ Thus, constant occurrences of movement disorders can be important factors to the development of TMD, since severe cases of movement disorders can cause jaw pain, dysarthria, dental, and temporomandibular traumas. ${ }^{28}$

Blocking the dopamine $\mathrm{D}_{2}$ receptor with the use of antipsychotic agents is the main cause of drug-induced movement disorders. ${ }^{5}$ Also called "extrapyramidal symptoms," these involuntary drug-induced motor symptoms are associated with the potency of the drug to provoke $\mathrm{D}_{2}$ receptor blockade in the nigrostriatal system. ${ }^{28}$ Such extrapyramidal effects include akathisia, dyskinesia, dystonia, and parkinsonism. Depending on the time of exposure to the drug administered, extrapyramidal effects also can be classified as: acute, occurring within hours or days, usually after initiation of the drug or, less commonly, when changing doses; subacute, occurring weeks after use, emerging during treatment, but usually ceasing when the drug is withdrawn; and tardive, occurring months after use, emerging typically after chronic treatment, and often not remitting after drug withdrawal. . $^{29,30}$

Antipsychotic drugs are the mainstay for the treatment of schizophrenia. Commonly, atypical antipsychotics produce less extrapyramidal symptoms than typical or conventional antipsychotic agents. ${ }^{5,31}$ This is because atypical antipsychotic drugs produce an effect on psychotic symptoms at lower doses than those required to induce extrapyramidal symptoms. ${ }^{29}$

In a meta-analysis of clinical improvement associated with the dopamine $\mathrm{D}_{2}$ receptor blockade by antipsychotic agents, it was observed that $80 \%$ of $\mathrm{D}_{2}$ receptor occupancy seems to be the critical cutoff point for the therapeutic effects of antipsychotic agents. The $\mathrm{D}_{2}$ receptor occupancy above $80 \%$ is associated with the presence of extrapyramidal symptoms with little gain in clinical improvement. ${ }^{32}$

Effects of antipsychotic agents in the long term can lead to oral dyskinesia and contribute to muscle stiffness, degenerative changes of the temporomandibular joint, mucosal lesions, and damage to teeth and dental prostheses., ${ }^{2,5}$ Such cases, designed as tardive dyskinesia, can appear later or as soon as in the first six weeks after the onset of an antipsychotic agent. Administration of antipsychotic drugs must be monitored; once advanced, cases of tardive dyskinesia can be difficult to reverse. It is estimated that even after discontinuation of antipsychotic medication, about $50 \%$ of patients continue to experience chronic symptoms of tardive dyskinesia. ${ }^{3}$

The specific pathophysiology of tardive dyskinesia, however, is not clear. It is speculated that there are possible mechanisms involving the hypersensitivity postsynaptic dopamine receptor, abnormalities related to the striatal neurons which utilize gamma-aminobutyric acid (GABA), and degeneration of striatal cholinergic interneurons. ${ }^{33}$

Anticholinergic agents are commonly administered to treat or prevent extrapyramidal symptoms induced by antipsychotic agents. However, the use of anticholinergics in patients with schizophrenia has been associated with central and peripheral nervous system side effects. ${ }^{4}$ The most distressing peripheral adverse effects are dry mouth, urinary disturbances, and constipation, while the most marked central adverse effects are the worsening of tardive dyskinesia, delirium, and decreased cognitive performance in executive functions, memory, learning, and attention. ${ }^{4,34}$

Benzodiazepines can also be used alternatively to minimize cases of dystonia in patients with schizophrenia. They are sedative-hypnotic agents which bind to the $\mathrm{GABA}_{\mathrm{A}}$ receptors, activating the GABAergic neurons that operate on the inhibitory central nervous system. Thus, benzodiazepine agents tend to reduce the communication between neurons and promote muscle relaxation. ${ }^{28}$

On some occasions, benzodiazepine drugs can produce mild respiratory suppression and blunt arousal response, 
increasing the severity of obstructive sleep apnea cases. Thus, the administration of benzodiazepines in schizophrenic patients has a very high risk, because these patients seem to have more cases of sleep apneia than subjects with other psychiatric conditions. ${ }^{35}$ Furthermore, the use of benzodiazepine agents in patients with schizophrenia has been associated with poor performance on tasks of working memory and motor speed. ${ }^{36}$

Medications, such as reserpine and tetrabenazine, produce improvement in patients with generalized dystonia, since they are drugs that deplete presynaptic dopamine and have weak blocking properties of the dopamine receptors. ${ }^{28}$ Compared with reserpine, tetrabenazine has a faster onset of action and fewer peripheral effects on the catecholamine depletion. ${ }^{33}$ This is because reserpine is an irreversible inhibitor of human vesicular monoamine transporter 2 , while tetrabenazine is a reversible inhibitor. ${ }^{37}$

\section{Pain, stress, and schizophrenia}

Pain and stress have a negative influence on the general health and quality of life, reflecting on social and occupational functioning, as well as on affective balance and cognitive development of individuals. ${ }^{38}$ In the general population, pain is the most common reason for seeking treatment for TMD. ${ }^{2}$ Therefore, chronic pain is an important symptom that describes TMD like psychophysiological disorders of the central nervous system that modulate neuroendocrine and physiological responses arising from emotional and physical stress. ${ }^{24}$ Thus, the reduction or absence of pain can contribute to TMD not being diagnosed and treated, which can lead to serious long-term consequences. ${ }^{2}$

Pathophysiological and psychological components of pain phenomenon are often difficult to interpret. ${ }^{39}$ Multiple regulatory domains that are associated with the pain seem to integrate the action from sensory, autonomic, inflammatory and psychological functions. This set of actions that involve the central nervous system, has as main objective the maintenance of homeostasis. Then, the experience of pain can be modulated by different pathways; it takes into account the participation of multiple compensatory systems. ${ }^{40}$

Stress, anxiety, and depression can alter nociceptive impulses from the central nervous system and the release of neurotransmitters, which may lead to changes in pain threshold. ${ }^{38}$ In turn, muscle pain can also generate a vicious cycle involving high levels of basal muscle tone, low levels of maximum voluntary contraction, and poor neuromuscular control, what may lead to increased joint stress, rigidity, low variability of movement, and more pain. ${ }^{41}$
Pain in TMD is located in the jaw, temporomandibular joint, and masticatory muscles. ${ }^{16}$ Stress, in turn, has been shown to be associated with repeated behaviors such as jaw clenching and teeth grinding that tend to contribute to the triggering of temporomandibular problems. ${ }^{42}$ Therefore, it is suggested that there may be differences in the psychological profile of patients with muscle complaints and joint complaints. Apparently, patients with muscle complaints are more sensitive to pain and more prone to psychological stress than patients with joint complaints. ${ }^{43}$

In schizophrenia, it is common to have signs of TMD without pain symptomatology. ${ }^{11}$ The prevalence of reduction or absent reaction to pain is reported as being 37\%-91\% in individuals with schizophrenia who have suffered from various painful medical conditions, like appendix rupture, bowel perforation, and peritonitis, among others. ${ }^{44}$

Schizophrenic patients seem to be less sensitive to bodily discomfort and tend to remain for long periods in contracted or uncomfortable positions without manifesting any complaint of pain. ${ }^{8}$ Controversially, they may also exhibit bizarre sensory complaints, such as a feeling of "pins and needles", due to a disorder of body awareness called "coenesthesia". ${ }^{45}$

Several neurologic deficits are observed in many subjects with schizophrenia. These are classified as soft neurological signs and hard neurological signs. Soft neurological signs are designed as nonfocal neurological indicators that involve the presence of primitive reflexes, sensory integration failures, motor coordination failures, and motor sequencing failures. Hard neurological signs are specific neurological damages that include hypoalgesia, loss of olfactory function, and oculomotor abnormalities. ${ }^{46}$ However, the accurate pathophysiology of hypoalgesia in schizophrenia is very poorly understood, despite its being strongly associated with increased morbidity and mortality among patients. ${ }^{26}$

It is speculated that hypoalgesia in schizophrenia is a nonspecific dulling response to pain, and it can be considered one of several manifestations of blunted response to the primary bodily sensations. This is due to the fact that people with schizophrenia often have a blunted response not only to pain, but to pleasure and to basic emotions. ${ }^{44}$

Some individuals with schizophrenia have reported experiencing pain, most commonly headache. ${ }^{11}$ This discomfort is presented as one of the most common symptoms of TMD. ${ }^{16}$ Also, symptoms, such as kinesthetic delusions, hypochondria, strange thoughts, or delusions related to internal organs, such as those described in Cotard's syndrome, suggest failure of pain perception in schizophrenia. ${ }^{8}$ 
Apparently, individuals with schizophrenia have a response profile to pain that is unique - characterized by increased sensitivity to acute pain and a reduced sensitivity to prolonged pain. In addition, the intensity of the nociceptive threshold in patients with schizophrenia seems to be different than in control populations. Schizophrenic patients have sensation threshold, pain threshold and pain tolerance significantly higher than healthy controls of the same age and sex..$^{25}$ Also, antipsychotic medications seem to have an effect on the perception of pain, further contributing to a reduced sensitivity to physical pain presented by individuals with schizophrenia. ${ }^{2}$ Nevertheless, a meta-analysis on hypoalgesia in schizophrenia concluded that the decrease in pain sensitivity in schizophrenia is not necessarily an artifact of antipsychotic medications. ${ }^{44}$

\section{Implications for clinical practice}

Schizophrenic patients with TMD tend to have more dental attrition, abrasion, and erosion. This is because TMD are often associated with parafunctional activities, such as bruxism. ${ }^{2}$ In some cases, patients with schizophrenia can have bilateral or unilateral dislocation of the jaw, lesions on the lips and tongue, due to generalized muscle tension combined with the side effects of psychotropic medications. ${ }^{2,28}$ Other complications can include speech impairment, dysphasia, chewing difficulties, and inadequate food intake. ${ }^{47}$

Particularly, dislocation of the jaw makes the patient unable to close his mouth because the condyle is displaced anteriorly to the articular eminence and fixed in that position by muscle spasm. The complete dislocation of the jaw arises due to the separation of the articular surface, while an incomplete dislocation of the jaw occurs when the joint surfaces still maintain partial contact, and the condyle is able to return to the glenoid fossa voluntarily or by self-manipulation. ${ }^{48}$

Many patients with TMD usually tend to complain of headaches, neck pain, pain in the face, and ear pain. Other associated complaints with TMD may include tinnitus, ear fullness, and perceived hearing loss. ${ }^{49}$ The co-occurring tinnitus and TMD in schizophrenic patients can be confounded with auditory hallucinations. The nondifferentiation of such complaints may influence the judgment of the true mental status of the patient. ${ }^{50}$

\section{Treatment of temporomandibular problems in schizophrenia}

Treatment of TMD requires different procedures that must take into account the type of dysfunction. For this reason, treatment plans for TMD must rely on an accurate diagnosis that meets the needs of each patient and allows specific results to be achieved. ${ }^{24}$

In patients with schizophrenia, the peculiarities of their mental illness may limit some management of TMD that requires the major cooperation of the subject. In these cases, the administration of an atypical antipsychotic agent seems to be most advantageous for the treatment of schizophrenic patients with TMD. The atypical antipsychotics tend to improve the negative symptoms of schizophrenia, allowing these patients to adhere to treatment. ${ }^{6}$

The most appropriate management model for patients with TMD is multidisciplinary. Functional improvement promotion in patients with TMD must involve physical and behavioral treatments, which may include actions of education and self-care, physical therapy, pharmacotherapy, cognitive, and behavioral intervention, among others. ${ }^{49}$

The great majority of TMD cases can be treated by noninvasive, nonsurgical, and reversible interventions. ${ }^{16}$ Each proposal for therapy and professional practice included in a TMD treatment plan must take into account the maximization of benefits and minimal costs in terms of price, time, and adverse effects. ${ }^{17}$

\section{Conclusion}

A great challenge is observed in the diagnosis and the treatment of TMD in psychiatric patients with severe problems, especially in those with schizophrenia. This is because such patients are exposed to a wide variety of medications that can contribute to the increased occurrence of TMD.

There is a need for careful clinical approaches in the evaluation of the signs and symptoms of TMD in subjects with schizophrenia, as it is done in relation to individuals with orofacial pain without major psychiatric disorders. In general, attention to physical illness in psychotic individuals is not sometimes valued and prioritized by health professionals.

Patients with schizophrenia tend to have little complaint of pain, a symptom that is commonly signaled by individuals suffering from TMD, and this can be a crucial factor for the negligence of care. Clinical strategies related to oral health care and preventive measures for alleviating the emotional stress can be a good way to reduce the incidence of TMD in this population.

\section{Disclosure}

The authors have no conflicts of interest to declare in this work.

\section{References}

1. Clark DB. Dental care for the patient with schizophrenia. Canadian Journal of Dental Hygiene. 2008;42(1):17-24. 
2. Gurbuz O, Alatas G, Kurt E. Prevalence of temporomandibular disorder signs in patients with schizophrenia. J Oral Rehabil. 2009;36(12): 864-871.

3. Reifsnyder JW, Tettambel MA. Conservative approach to tardive dyskinesia-induced neck and upper back pain. J Am Osteopath Assoc. 2013;113(8):636-639.

4. Ogino S, Miyamoto S, Miyake N, Yamaguchi N. Benefits and limits of anticholinergic use in schizophrenia: Focusing on its effect on cognitive function. Psychiatry Clin Neurosci. 2014;68(1):37-49.

5. Weiss A, Movahed R, Dym H. Schizophrenia: current therapy and review. J Oral Maxillofac Surg. 2011;69(1):192-198.

6. Tanasiewicz M, Skucha-Nowak M, Greń A, Hese RT, Twardawa H, Gorczyca P. Analysis of State of the Oral Cavity in Schizophrenic Inpatients Treated with Atypical and Classical Neuroleptics. Adv Clin Exp Med. 2011;20(2):187-197.

7. Matevosyan NR. Oral health of adults with serious mental illnesses: a review. Community Ment Health J. 2010;46(6):553-562.

8. Bonnot O, Anderson GM, Cohen D, Willer JC, Tordjman S. Are patients with schizophrenia insensitive to pain? A reconsideration of the question. Clin J Pain. 2009;25(3):244-252.

9. Velasco-Ortega E, Monsalve-Guil L, Velasco-Ponferrada C, Medel-Soteras R, Segura-Egea JJ. Temporomandibular disorders among schizophrenic patients. A case-control study. Med Oral Patol Oral Cir Bucal. 2005;10(4):315-322. Spanish [with English abstract].

10. Lévesque M, Potvin S, Marchand S, et al. Pain perception in schizophrenia: evidence of a specific pain response profile. Pain Med. 2012;13(12):1571-1579.

11. Dworkin RH. Pain insensitivity in schizophrenia: a neglected phenomenon and some implications. Schizophr Bull. 1994;20(2):235-248.

12. Salmos-Brito JA, de Menezes RF, Teixeira CE, et al. Evaluation of lowlevel laser therapy in patients with acute and chronic temporomandibular disorders. Lasers Med Sci. 2013;28(1):57-64.

13. Yin CS, Lee YJ, Lee YJ. Neurological influences of the temporomandibular joint. J Bodyw Mov Ther. 2007;11(4):285-294.

14. Aditya A, Lele S, Aditya P. Prevalence of symptoms associated with temporomandibular disorders in patients with psychosocial disorders. Journal of International Dental and Medical Research. 2012;5(1):26-29.

15. McNeely ML, Armijo Olivo S, Magee DJ. A systematic review of the effectiveness of physical therapy interventions for temporomandibular disorders. Phys Ther. 2006;86(5):710-725.

16. Scrivani SJ, Keith DA, Kaban LB. Temporomandibular disorders. N Engl J Med. 2008;359(25):2693-2705.

17. Wright EF. Manual of Temporomandibular Disorders. 2nd ed. Oxford, UK: Wiley-Blackwell; 2009.

18. Cruccu G, Frisardi G, Pauletti G, Romaniello A, Manfredi M. Excitability of the central masticatory pathways in patients with painful temporomandibular disorders. Pain. 1997;73(3):447-454.

19. Laskin DM. Temporomandibular disorders: a term past its time? J Am Dent Assoc. 2008;139(2):124-128.

20. Paesani D, Westesson PL, Hatala M, Tallents RH, Kurita K. Prevalence of temporomandibular joint internal derangement in patients with craniomandibular disorders. Am J Orthod Dentofacial Orthop. 1992;101(1): 41-47.

21. Kight M, Gatchel RJ, Wesley L. Temporomandibular disorders: evidence for significant overlap with psychopathology. Health Psychol. 1999;18(2):177-182.

22. Al-Jundi MA, John MT, Setz JM, Szentpétery A, Kuss O. Meta-analysis of treatment need for temporomandibular disorders in adult nonpatients. J Orofac Pain. 2008;22(2):97-107.

23. Shibukawa Y, Ishikawa T, Kato Y, et al. Cerebral cortical dysfunction in patients with temporomandibular disorders in association with jaw movement observation. Pain. 2007;128(1-2):180-188.

24. Goldstein BH. Temporomandibular disorders: a review of current understanding. Oral Surg Oral Med Oral Pathol Oral Radiol Endod. 1999;88(4):379-385.
25. Winocur E, Hermesh H, Littner D, Shiloh R, Peleg L, Eli I. Signs of bruxism and temporomandibular disorders among psychiatric patients. Oral Surg Oral Med Oral Pathol Oral Radiol Endod. 2007;103(1): 60-63.

26. Singh MK, Giles LL, Nasrallah HA. Pain insensitivity in schizophrenia: trait or state marker? J Psychiatr Pract. 2006;12(2):90-102.

27. Balasubramaniam R, Ram S. Orofacial movement disorders. Oral Maxillofac Surg Clin North Am. 2008;20(2):273-285, vii.

28. Eken C, Güler V, Koparan C, Çicek M. Temporomandibular joint dislocation due to haloperidol induced acute dystonia: a case report and review of the literature. Erciyes Medical Journal. 2009;1: $10-13$.

29. Claxton KL, Chen JJ, Swope DM. Drug-Induced Movement Disorders. J Pharm Pract. 2007;20(6):415-429.

30. Saifee TA, Edwards MJ. Tardive movement disorders: a practical approach. Pract Neurol. 2011;11(6):341-348.

31. de Araújo AN, de Sena EP, de Oliveira IR, Juruena MF. Antipsychotic agents: efficacy and safety in schizophrenia. Drug Healthc Patient Saf. 2012;4:173-180.

32. Yilmaz Z, Zai CC, Hwang R, et al. Antipsychotics, dopamine $\mathrm{D}_{2}$ receptor occupancy and clinical improvement in schizophrenia: a meta-analysis. Schizophr Res. 2012;140(1-3):214-220.

33. Jankelowitz SK. Treatment of neurolept-induced tardive dyskinesia. Neuropsychiatr Dis Treat. 2013;9:1371-1380.

34. Ogino S, Miyamoto S, Tenjin T, et al. Effects of discontinuation of long-term biperiden use on cognitive function and quality of life in schizophrenia. Prog Neuropsychopharmacol Biol Psychiatry. 2011;35(1): 78-83.

35. Lin WC, Winkelman JW. Obstructive sleep apnea and severe mental illness: evolution and consequences. Curr Psychiatry Rep. 2012;14(5): 503-510.

36. Kishi T, Moriwaki M, Kawashima K, et al. Investigation of clinical factors influencing cognitive function in Japanese schizophrenia. Neurosci Res. 2010;66(4):340-344.

37. Chen JJ, Ondo WG, Dashtipour K, Swope DM. Tetrabenazine for the treatment of hyperkinetic movement disorders: a review of the literature. Clin Ther. 2012;34(7):1487-1504.

38. Resende CM, Alves AC, Coelho LT, Alchieri JC, Roncalli AG, Barbosa GA. Quality of life and general health in patients with temporomandibular disorders. Braz Oral Res. 2013;27(2):116-121.

39. Marazziti D, Mungai F, Vivarelli L, Presta S, Dell'Osso B. Pain and psychiatry: a critical analysis and pharmacological review. Clin Pract Epidemiol Ment Health. 2006;2:31.

40. Chen H, Nackley A, Miller V, Diatchenko L, Maixner W. Multisystem dysregulation in painful temporomandibular disorders. J Pain. 2013; 14(9):983-996.

41. Hodges PW, Tucker K. Moving differently in pain: a new theory to explain the adaptation to pain. Pain. 2011;152(Suppl 3):S90-S98.

42. Reneker J, Paz J, Petrosino C, Cook C. Diagnostic accuracy of clinical tests and signs of temporomandibular joint disorders: a systematic review of the literature. J Orthop Sports Phys Ther. 2011;41(6):408-416.

43. Rollman GB, Gillespie JM. The role of psychosocial factors in temporomandibular disorders. Curr Rev Pain. 2000;4(1):71-81.

44. Potvin S, Marchand S. Hypoalgesia in schizophrenia is independent of antipsychotic drugs: a systematic quantitative review of experimental studies. Pain. 2008;138(1):70-78.

45. Jochum T, Letzsch A, Greiner W, Wagner G, Sauer H, Bär KJ. Influence of antipsychotic medication on pain perception in schizophrenia. Psychiatry Res. 2006;142(2-3):151-156.

46. Tandon R, Nasrallah HA, Keshavan MS. Schizophrenia, "just the facts" 4. Clinical features and conceptualization. Schizophr Res. 2009;110(1-3):1-23.

47. Pekkan G, Kilicoglu A, Algin DI. Treatment of a tardive dyskinesia patient with temporomandibular disorder: a case report. J Orofac Pain. 2010;24(2):212-216 
48. Undt G, Weichselbraun A, Wagner A, Kermer C, Rasse M. Recurrent mandibular dislocation under neuroleptic drug therapy, treated by bilateral eminectomy. J Craniomaxillofac Surg. 1996;24(3):184-188.

49. McNeill C. Management of temporomandibular disorders: concepts and controversies. J Prosthet Dent. 1997;77(5):510-522.
50. Nam EC. Is it necessary to differentiate tinnitus from auditory hallucination in schizophrenic patients? J Laryngol Otol. 2005;119(5): $352-355$.

Drug, Healthcare and Patient Safety

\section{Publish your work in this journal}

Drug, Healthcare and Patient Safety is an international, peer-reviewed open-access journal exploring patient safety issues in the healthcare continuum from diagnostic and screening interventions through to treatment, drug therapy and surgery. The journal is characterized by the rapid reporting of reviews, original research, clinical, epidemiological and

\section{Dovepress}

post-marketing surveillance studies, risk management, health literacy and educational programs across all areas of healthcare delivery. The manuscript management system is completely online and includes a very quick and fair peer-review system. Visit http://www.dovepress.com/ testimonials.php to read real quotes from published authors.

Submit your manuscript here: http://www.dovepress.com/drug-healthcare-and-patient-safety-journal 